\title{
Evaluer la densité des ligneux par la méthode des distances ? Un problème
}

\author{
Ph. Daget ${ }^{1}$ A. Ickowicz ${ }^{2}$ M. Mbaye ${ }^{3}$
}

\begin{abstract}
Mots-clés
Pâturages - Mesure - M éthode - Echantillonnage - Végétation - Densité Plante ligneuse - Zone tropicale.
\end{abstract}

\begin{abstract}
Résumé
L'estimation de la densité des ligneux dans les savanes est une étape souvent nécessaire dans l'étude de la production de ces pâturages. L'expérience a montré que les observations directes par dénombrement donnaient souvent des valeurs différentes de celles qui sont obtenues par les méthodes Ppi (plus proche individu) et Q cp (quadrant centré sur un point) de la Plotless method. Les auteurs montrent que ces divergences sont liées à l'hétérogénéité de la répartition des individus dans l'espace.
\end{abstract}

\section{INTRODUCTION}

La méthode d'étude de la végétation proposée par Clark et Evans (2) est connue sous le nom de Plotless method, mais aussi de " méthode du quadrant centré sur un point » (Qcp) ou encore de « méthode du plus proche individu » (Ppi). Elle consiste à répartir dans l'unité de végétation à échantillonner 30 à 100 points et à mesurer la distance séparant chacun d'eux de l'individu le plus proche dans le cas de la méthode Ppi ou des quatre individus les plus proches dans celui de la méthode Qcp. La distance moyenne m* est ensuite calculée ; si la végétation est homogène, la densité — c'est-à-dire le nombre d'individus par unité de surface - est donnée par deux formules :

$$
\mathrm{d}=\frac{1}{4 \mathrm{~m}^{2}} \text { (méthode Ppi) ou } \mathrm{d}=\frac{1}{\mathrm{~m}^{2}} \text { (méthode Qcp) }
$$

Cette méthode a été très tôt transposée à l'étude de la strate haute dans les steppes et savanes arborées des régions tropicales (13, 14). Non sans difficultés puisque, dans un travail récent, Ickowicz (11) se heurte encore à des divergences déjà signalées $(9,15,16)$ pouvant être importantes entre la densité calculée de cette manière et la «densité vraie » obtenue par le dénombrement complet des individus dans l'unité de végétation. Cette divergence pose un problème sur lequel nous allons insister ici.

1. Cirad-emvt/Cnrs, Campus international de Baillarguet, TA 30/F, 34398 Montpellier Cedex 5, France ; E-mail : philippe.daget@cirad.fr

2. Cirad-emvt/Isra-Lnerv, BP 2057, Dakar, Sénégal

E-mail : ickowicz@telecomplus.sn

3. Isra-Lnerv, BP 2057, Dakar, Sénégal

\section{APPROCHETHEORIQUE}

\section{Distribution uniforme}

Tout d'abord, il faut bien voir que cette méthode ne peut être appliquée qu'à des végétaux aisément dénombrables et faciles à distinguer, donc pas aux graminées ni aux espèces constituant le fond du tapis végétal dans des formations denses : elle n'est pas générale. Mais les auteurs évoqués l'utilisaient pour caractériser la strate arborée de savanes boisées et non pas les strates basses ; ce n'est donc pas sur ce point que le problème se pose.

Ensuite, il faut que la végétation soit « homogène ». Souvent ce terme est pris comme synonyme de « répartie au hasard », ce qui en soit ne signifie pas grand-chose. Il faut spécifier selon quelle loi cette répartition est faite (ou supposée faite) ; le plus souvent il s'agit d'une répartition selon la loi uniforme, ou plus brièvement de répartition uniforme. Parfois confondue avec la répartition régulière, la répartition uniforme est telle que les individus de l'espèce examinée ont une probabilité d'occurrence égale en toute unité de surface de la station (6). Il est clair qu'une végétation peut être homogène pour une espèce et ne pas l'être pour une autre. Il existe des méthodes pour analyser les écarts à l'homogénéité d'une végétation, mais au plan phytosociologique cette homogénéité est, le plus souvent, appréciée visuellement $(1,3,10)$. C'est à ce niveau que les problèmes se posent.

\section{SIM U LATIO N S}

Trois « végétations » ont été simulées sur ordinateur, avec 250 individus répartis dans un carré de $100 \mathrm{~m}$ de côté. Par construction donc, la densité est de 250/10 000 =0,025. Chacune de ces végéta- 
tions a été échantillonnée selon la Plotless method au moyen de 100 points régulièrement disposés le long de la diagonale ascendante du carré. Une application «inverse » de la définition de la densité permet d'obtenir la distance moyenne théorique attendue, celle qui correspondrait à une distribution uniforme rigoureuse :

$$
0,025=\frac{1}{4 \mathrm{~m}^{2}} \text { d'où } \mathrm{m}=\sqrt{10}=3,1623
$$

- La première population artificielle est obtenue par un tirage aléatoire des positions des 250 pieds. La distance moyenne telle qu'elle a été définie plus haut est $\mathrm{m}^{*}=3,6556$ avec une déviation standard $\mathrm{s}=1,452$. La densité appréciée selon la formule précédente est $d=0,0187$, valeur très sensiblement plus faible que la valeur vraie. La comparaison de la moyenne calculée avec la moyenne attendue par le test $\mathbf{t}$ donne :

$$
\mathrm{t}=\frac{3,6556-3,1623}{\frac{1,452}{10}}=\frac{0,4933}{0,1452}=3,397
$$

très nettement supérieure à la valeur liminaire au seuil de 1 p. 100 $(2,326)$. Il y a donc une différence significative entre $\mathrm{m}^{*}$ et sa valeur théorique qui explique la trop faible densité calculée. Pourtant, à l'œil, la répartition des points sur la figure 1 ne semble pas présenter de défaut majeur.

- Dans une seconde population artificielle obtenue, comme la précédente, par des tirages aléatoires, les résultats sont les suivants :

$$
\begin{array}{ll}
\mathrm{m}^{*}=3,1856 & \mathrm{~s}=1,83 \\
\mathrm{t}=0,127 & \mathrm{~d}=0,0246
\end{array}
$$

La différence entre la moyenne calculée et la moyenne attendue n'est pas significative et la densité mesurée est presque égale à la densité théorique.

- Une population agrégée a été construite en tirant 25 points selon la loi uniforme dans le même espace, puis 9 points selon la loi uniforme autour de chacun d'eux, mais dans un espace dix fois plus petit (figure 2). Les résultats obtenus sont les suivants :

$$
\begin{array}{ll}
\mathrm{m}^{*}=4,3945 & \mathrm{~s}=2,0597 \\
\mathrm{t}=6,156 & \mathrm{~d}=0,0129
\end{array}
$$

La différence entre la moyenne calculée et la moyenne attendue est hautement significative $(\mathrm{p}<0,001)$ et la densité calculée est voisine de la moitié de la valeur vraie.

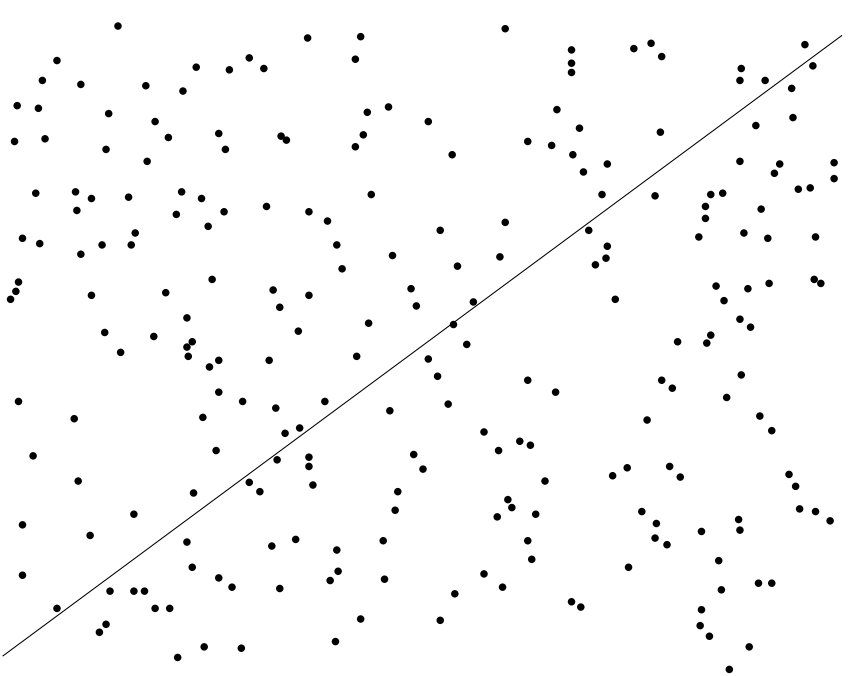

Figure 1 : population artificielle obtenue par tirage des coordonnées des points suivant une loi uniforme.

\section{Commentaires}

Le premier et surtout le troisième cas permettent de retrouver les divergences signalées et les modèles présentés conduisent à la réponse déjà formulée par Piélou (12) : "The formula is an estimator of the density only when the population has a random process (c'est à dire une répartition uniforme) so it can be used only if we can safely assume that a pattern is indeed random. " Donc la mesure de la densité est bonne dans le deuxième cas parce qu'il présente une répartition uniforme, elle est médiocre dans le premier cas qui s'en écarte faiblement et mauvaise dans le troisième cas parce qu'il n'est absolument pas uniforme. Elle est mauvaise dans les steppes et les savanes, comme elle le serait dans la plupart des végétations spontanées car les individus des diverses espèces n'y sont pas répartis de manière uniforme dans la plupart des cas (7).

Sous le nom de coefficient de variation, le rapport de la variance à la moyenne est souvent pris comme un indice de la dispersion des données :

$$
\mathrm{I}=\frac{\mathrm{VAR}}{\mathrm{MOY}}
$$

mais on utilise aussi (14) :

$$
\mathrm{d}=(\mathrm{k}-1) \mathrm{I}=(\mathrm{k}-1) \frac{\mathrm{VAR}}{\mathrm{MOY}}
$$

et (15) :

$$
\mathrm{I}^{*}=\mathrm{I}-1
$$

Lorsque les individus sont distribués de manière homogène, l'indice I n'est pas significativement différent de 1 , l'indice I* de 0 et $\mathbf{d}$ de $\mathrm{k}-1$; pour cela, il faut que la statistique

$$
\mathrm{x}^{2}=\frac{\mathrm{d}}{(\mathrm{k}-1)}
$$

ne soit pas significativement différente du $\chi^{2}$ à $\mathrm{k}$ - $1 \boldsymbol{d} \boldsymbol{d l}(5)$, mais cela ne suffit pas. C'est ainsi que différentes itérations de la modélisation de populations agrégées ont donné des indices très élevés — supérieurs à 5 - mais aussi des indices voisins de 1 .

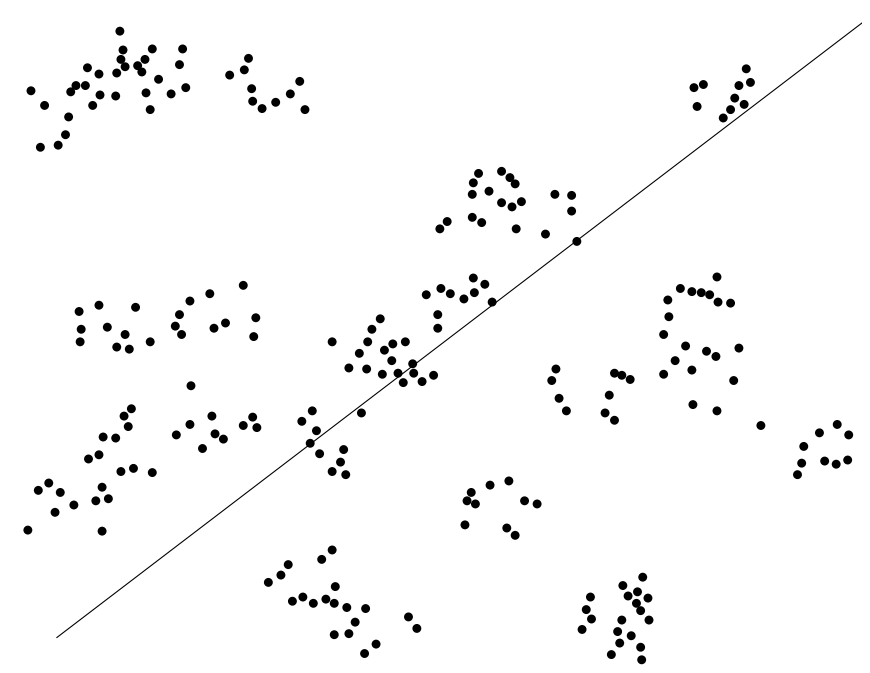

Figure 2 : population artificielle obtenue par tirage des coordonnées des points suivant une loi agrégative forte. 


\section{MISE EN PRATIQ UE}

\section{Matériel et Méthodes}

Une application pratique est proposée sur des mesures effectuées en Haute Casamance dans la région de Kolda, au Sénégal. Dans cette végétation de savane arbustive, deux lignes perpendiculaires de $120 \mathrm{~m}$ se croisant en leur centre ont été mises en place ; sur ces lignes, un point d'observation a été pris tous les $10 \mathrm{~m}$, plus un point central, soit un total de 25 points d'observation. De chaque point, la distance au pied le plus proche a été mesurée en mètres, en retenant successivement les arbres, les arbustes et les buissons, sans tenir compte de leur nature spécifique.

\section{Résultats}

Arbres

Distances

$\begin{array}{lllllll}5,00 & 1,05 & 3,80 & 6,95 & 5,25 & 6,25 & 3,80 \\ 1,00 & 2,26 & 1,04 & 2,96 & 6,00 & 3,97 & 2,00 \\ 4,55 & 3,00 & 4,40 & 0,96 & 4,60 & 3,00 & 2,84 \\ 9,80 & 4,12 & 2,35 & 4,75 & & & \end{array}$

Statistiques sur les distances

$\begin{array}{lc}\text { Effectif } & 25 \\ \text { Maximum } & 9,80 \\ \text { Minimum } & 0,96 \\ \text { Ecart absolu } & 8,84 \\ \text { Moyenne } & 3,828 \\ \text { Variance } & 4,3579 \\ \text { Déviation standard } & 2,0876 \\ \text { Déviation moyenne } & 1,5763 \\ \text { Médiane } & 3,80\end{array}$

Effectif réel sur un hectare : 140

Densité vraie : 0,014

Distance moyenne théorique : 4,2277

Distance moyenne observée : 3,828

Test $t$ sur les distances :

$$
\mathrm{t}=\frac{3,828-4,2277}{\frac{2,08757}{5}}=0,96
$$

$\mathrm{t}<1,96$ donc la différence observée n'est pas significative et par suite les individus arborés peuvent être considérés comme répartis uniformément, la formule de calcul de la densité était applicable, elle donne $\mathrm{d}=0,01706$.

\section{Arbustes}

Distances

$\begin{array}{lllllll}1,30 & 2,42 & 2,46 & 2,50 & 2,92 & 3,70 & 3,20 \\ 8,72 & 1,40 & 1,05 & 0,66 & 2,10 & 2,60 & 1,66 \\ 1,05 & 0,84 & 2,15 & 3,55 & 2,20 & 0,50 & 1,90 \\ 8,10 & 2,34 & 2,67 & 4,50 & & & \end{array}$

Statistiques sur les distances

$\begin{array}{lc}\text { Effectif } & 25 \\ \text { Maximum } & 8,72 \\ \text { Minimum } & 0,50 \\ \text { Ecart absolu } & 8,22 \\ \text { Moyenne } & 2,4196 \\ \text { Variance } & 2,6834 \\ \text { Déviation standard } & 1,6381 \\ \text { Déviation moyenne } & 1,01 \\ \text { Médiane } & 2,20\end{array}$

Effectif réel sur un hectare : 797

Densité vraie : 0,0797
Distance moyenne théorique : 1,7711

Distance moyenne observée : 2,4196

Test $t$ sur les distances :

$$
\mathrm{t}=\frac{2,4196-1,77109}{\frac{1,6381}{5}}=1,9795
$$

t >1,96 donc la différence observée est (tout juste) significative et par suite les individus arbustifs ne peuvent être considérés comme répartis uniformément, la formule de calcul de la densité n'était pas applicable, en fait elle donne $\mathrm{d}=0,0427$.

\section{Buissons}

Distances

$\begin{array}{lllllll}0,05 & 0,11 & 0,10 & 0,64 & 0,52 & 0,40 & 0,40 \\ 0,50 & 0,20 & 0,50 & 0,44 & 0,20 & 0,40 & 0,27 \\ 0,05 & 0,35 & 0,45 & 0,65 & 0,42 & 0,05 & 0,30 \\ 0,40 & 0,45 & 0,30 & 0,05 & & & \end{array}$

Statistiques sur les distances

$\begin{array}{lc}\text { Effectif } & 25 \\ \text { Maximum } & 0,65 \\ \text { Minimum } & 0,05 \\ \text { Ecart absolu } & 0,60 \\ \text { Moyenne } & 0,328 \\ \text { Variance } & 0,3398 \\ \text { Déviation standard } & 0,1843 \\ \text { Déviation moyenne } & 0,1542 \\ \text { Médiane } & 0,40\end{array}$

Effectif réel sur un hectare : 13732

Densité vraie : 1,373

Distance moyenne théorique : 0,4267

Distance moyenne observée : 0,328

Test $t$ sur les distances :

$$
\mathrm{t}=\frac{0,328-0,427}{\frac{0,1843}{5}}=-2,685
$$

$|\mathrm{t}|>$ 2,326 donc la différence observée est hautement significative et par suite les individus d'arbrisseaux ne peuvent pas du tout être considérés comme répartis uniformément, la formule de calcul de la densité n'était pas applicable, en fait elle donne $\mathrm{d}=2,324$, près du double de la densité vraie.

\section{Discussion}

Pour 25 valeurs, les limites du $\chi^{2}$ sont 42,98 et 10,86 au seuil de 99 p. 100 et de 36,42 et 13,85 au seuil de 95 p. 100 ; rapportés aux indices de dispersion, ils deviennent respectivement $1,791-0,453$ et $1,518-0,577$.

- pour les arbres, on a

$$
\mathrm{I}=\frac{0,034}{0,33}=0,102
$$

valeur supérieure à l'unité donc traduisant une surdispersion ; comprise entre 1,518 et 0,577 , cette surdispersion n'est pas significative ;

- pour les arbustes, avec

$$
\mathrm{I}=\frac{2,68}{2,42}=1,112
$$

les conclusions sont identiques ;

- pour les buissons,

$$
\mathrm{I}=\frac{0,034}{0,33}=0,102
$$

cette valeur inférieure au seuil le plus bas $(0,453)$ traduit une sous-dispersion hautement significative des individus. 
En définitive, les mesures de distances des deux premiers cas peuvent autoriser l'utilisation de la formule d'évaluation de la densité ; ce n'est pas possible pour les buissons et dans ce cas, il faut procéder par une autre approche.

La distance calculée à partir de la densité réelle dans le cas des arbustes est plus petite que la distance moyenne théorique ; cela devrait correspondre à une sous-dispersion alors que le calcul traduit une surdispersion. Ce fait tient à ce que le calcul porte sur les individus à proximité du dispositif et que l'évaluation directe porte sur tous les individus de l'hectare.

\section{- CON CLU SIO N}

Comme Cottam et coll. (4) l'avaient annoncé, les problèmes rencontrés dans l'utilisation de la Plotless method pour l'estimation de la densité de la végétation ont pour origine la structure irrégulière de la répartition des individus dans l'espace et les formules ne constituent pas « an estimator of the density only when the population has a random pattern » comme le dit Piélou (12). Mais sa conclusion «It is not possible in practice to estimate density of natural population of organism... distance measurement alone are not enough and we must also carry out quadrat sampling or complete count of population » est excessive et nous verrons dans quelques notes ultérieures comment il peut être possible d'éviter de compléter les «mesures » de la densité par l'examen exhaustif de quadrats (11). En pratique, après un échantillonnage par la Plotless method, une estimation de l'indice de dispersion et un simple test statistique permettent de porter un jugement sur la fiabilité de l'estimation de la densité pouvant en être déduite.

\section{BIBLIO GRAPHIE}

1. BRAU N-BLAN Q UET J., 1928. Pflanzensoziologie. Berlin, Allemagne Springer, $330 \mathrm{p}$.

2. CLARK P. EVANS F., 1954. Distance to nearest neighbour as a measure of spatial relationships in populations. Ecol., 35: 445-455.
3. CONTANDRIOPOULOS J., 1962. Recherche sur la flore endémique de la Corse et sur ses origines. Ann. fac. Sci. Marseille, 32 : 1-354.

4. COTTAM G., CURTIS J., HALE B., 1953. Some sampling characteristics of a population of randomly dispersed individuals. Ecol., 34: 741-757.

5. COX D., LEWIS P., 1969. L'analyse statistique des séries d'événements. Paris, France, Dunod, $272 \mathrm{p}$.

6. DAGET P., 1976. Répartition des présences dans une série d'unités d'échantillonage, application à l'analyse de l'homogénéité. Nat. Monspeliensia, 26 : 95-108.

7. DAGET P., 1978. Ecologie générale et prairie permanente, de la reflexion fondamentale à l'application. Thèse d'Etat, Université M ontpellier II, Montpellier, France, $208 \mathrm{p}$.

8. DAVID F., MOORE P., 1954. Notes on contagious distributions in plant populations. Ann. Bot., 18: 47-53.

9. GUERIN H., 1994. Valeur alimentaire des fourrages ligneux consommés par les ruminants en Afrique centrale et de l'Ouest. Maisons-Alfort, France, Cirad-emvt, $350 \mathrm{p}$.

10. GUIN OCHET M., 1974. Phytosociologie. Paris, France, Masson, $227 \mathrm{p}$.

11. ICKO WICZ A., 1995. Approche dynamique du bilan fourrager appliquée à des formations pastorales du Sahel tchadien. Thèse Doct., U niversité Paris XII, Paris, France, $475 \mathrm{p}$.

12. PIELOU E., 1980. Biogeography. New York, NY, USA, John Wiley, $351 \mathrm{p}$.

13. PO ISSO NET J., TO URE I., 1986. Eléments pour une étude qualitative et quantitative de la végétation du Ferlo sénégalais. In : Séminaire régional sur la dynamique et l'évolution des écosystèmes pastoraux. Dakar, Sénégal, Fapis/U nesco, $12 \mathrm{p}$.

14. SEN A., TOURIGNY J., SMITH G., 1974. On the transect sampling method. Biometrics., 30: 329-340.

15. TOURE I., GILLET H., 1989. Techniques d'inventaire des ligneux et d'estimation de la biomasse ligneuse appétée. In : Compte-rendu du séminaire régional sur les fourrages et l'alimentation des ruminants, Maisons-Alfort, France. Maisons-Alfort, France, lemvt, p. 251-263.16. WALKER B., 1970. An evaluation of eight methods of botanical analysis on grassland in Rhodesia. J. Appl. Ecol., 7: 403-416.

Reçu le 13.7.99, accepté le 24.2.00

\section{Summary}

D aget $\mathbf{P}$., Ickowicz A., M baye $\mathbf{M}$. Evaluation of the density of the ligneous by distance-methods? A problem

The estimation of ligneous density in savannah is a necessary step in the study of the production of these pasture lands. The experience showed that direct observations by counting often gave different values from those obtained with PPI and QCP of the Plotless method. The authors show that theses divergences are linked to the heterogeneity of the stems distribution in the space.

Key words: Pastures - Measurement - Methods - Sampling Vegetation - D ensity - W oody plants - Tropical zones.

\section{Resumen}

Daget $\mathbf{P}$., Ickowicz A., Mbaye M. ¿Evaluar la densidad de los leñosos mediante el método de las distancias? U n problema

La estimación de los leñosos en las sabanas es una etapa a menudo necesaria en el estudio de la producción de estos pastizales. La experiencia ha mostrado que las observaciones directas por conteo dan a menudo valores diferentes de los obtenidos mediante los métodos PPI (del individuo más cercano) y QCP (del cuadrante centrado sobre un punto) del Plotless method. Los autores demuestran que estas divergencias están ligadas a la heterogeneidad de la repartición de los individuos en el espacio.

Palabras clave: Pastizales - Medición - Métodos - Muestreo Vegetación - D ensidad - Plantas leñosas - Zona tropical. 